\title{
sciendo
}

\author{
BULGARIAN ACADEMY OF SCIENCES
}

CYBERNETICS AND INFORMATION TECHNOLOGIES • Volume 20, No 6

Special Issue on New Developments in Scalable Computing

Sofia $\bullet 2020$

Print ISSN: 1311-9702; Online ISSN: 1314-4081

DOI: $10.2478 /$ cait-2020-0067

\section{Geometrically Non-Linear Vibration of a Cantilever Interacting with Rarefied Gas Flow}

\author{
Kiril Shterev, Emil Manoach \\ Institute of Mechanics, Bulgarian Academy of Sciences, 1113 Sofia, Bulgaria \\ E-mails: kshterev@imbm.bas.bg e.manoach@imbm.bas.bg
}

\begin{abstract}
The work is devoted to study $2 D$ pressure driven rarefied gas flow in a microchannel having an elastic obstacle. The elastic obstacle is clamped at the bottom channel wall and its length is half of the channel height. The gas flow is simulated by Direct Simulation Monte Carlo (DSMC) method applying the advanced Simplified Bernoulli Trial (SBT) collision scheme. The elastic obstacle is modelled as geometrically nonlinear Euler Bernoulli beam. A reduced 3 modes reduction model of the beam is created. The influence of the gas flow on the beam vibration is studied, considering the linear and nonlinear beam theories.
\end{abstract}

Keywords: Fluid-Structure Interaction (FSI), rarefied gas, microchannel, DSMC, Euler-Bernoulli beam, pressure driven flow.

\section{Introduction}

One of the most important coupled problem in mechanics is the Fluid Structures Interaction (FSI). The interaction between different fluid flows and rigid or elastic structures can be observed in many technological applications in civil engineering, mechanical engineering, energetics, etc. Many aspects of the FSI are deeply considered in the Paidoussis' book [1].

In this book and in many others studies, problems related to large structures are treated. In the past decades, however, with the development of the microtechnologies, the problems of the FSI at micro level became very important. This fact leads to the increasing number of studies in this field. At micro level, the phenomena that arise in microfluidic differ essentially from the conventional flows at macroscales. In many microsystems as Micro-ElectroMechanical Systems (MEMS), micro machines, flow sensors, etc. the flow interacts with deformable structures. The design of such systems requires theoretical modelling of the dynamic behaviour of the flexible structures in their interaction with the fluid. 
Many studies considering the interaction of a fluid with structures at micro level are connected with the modelling of the FSI in Atomic Force Microscopes (AFM). In [2] a simulation of the behaviour of AFM operating in liquid was studied by a lumped-parameter model of the beam combined with a transient Navier-Stokes solver. The beam is modelled in a very simple way as a harmonic oscillator. For the analysis of the fluid-structure interaction, the drag force is treated as a linear function of the velocity, which allows the system to be simplified to the classical massdamper-spring system. The equations of motion have been solved simultaneously with the time-dependent flow field.

The behaviour of the cantilever in AFM is considered again in [3]. The effect of fluid loading is analysed by a systems/feedback approach. The thermal response is analysed as well.

Interesting results are presented in the works of $\mathrm{Gree} \mathrm{n}$ and $\mathrm{S}$ a der [4] and Cornelis, Van and Sader [5]. Authors have studied the influence of the fluid flow on frequency response functions of cantilevered beams immersed in viscous fluids. The linear Euler-Bernoulli beam theory is used to describe the structure behaviour. The influence of the fluid on the beam vibration is taken into account as a hydrodynamic force, which is added to another driving force. In [4] the thermal driving is considered while in [5] a thermal driving force and a driving force obtained from the solution of the linearized Navier-Stokes equation is considered. Flexural and torsional modes of vibrations are considered separately. The studies show that the higher order modes of the cantilever can be significantly affected by the induced three-dimensional nature of the flow field around the cantilever.

In [6] a FSI Algorithm is presented using compressible continuum fluid model, such that the equivalent of first-order slip velocity and temperature jump boundary conditions are achieved at fluid-solid boundaries. The FSI Algorithm uses a threedimensional, unsteady, continuum based Eulerian-Lagrangian methodology. The fluids are modelled using ICE (Implicit, Continuous fluid, Eulerian) model and solid materials are modelled by the Material-Point-Method (MPM). The transient, thermal/structural response of a damped-oscillatory three-dimensional finite cylinder subject to an impulsively started uniform, rarefied flow is studied.

The interaction between a microchannel with a deformable wall and the fluid that flows within it has been investigated experimentally and numerically fluidstructural in [7]. Water and sucrose syrup have been employed as working fluid in experiments while numerical calculations used incompressible continuum fluid model. Authors have compared displacement profiles of the small deformable region measured experimentally with that predicted using a two- and three-dimensional finite element model that incorporates coupling between the thin wall and the fluid. They report an excellent agreement between experiments and numerical results.

An attempt to study the interaction between an elastic structure and fluid flow by a strong coupling of the equations is made in [8]. By using the finite difference method, authors have developed a user program for a plate vibration that they have connected with 3D Flow commercial program. Then they have studied the dynamic behaviour of the MEMS diaphragm drop ejector. 
In [9] an approach similar to the one in [8] for FSI modelling is presented. Structural dynamics has been solved by a user defined finite element method solver coupled with the commercial Computational Fluid Dynamics (CFD) solver FLUENT based on a coupling approach, i.e., by allowing the information to be exchanged at the end of each time step. During the solution, the interface motion is transferred by using the moving mesh capability of the CFD solver.

In the references cited, the beam model is usually a simple one and does not include nonlinearities. The second and most important fact is that the interaction of rarefied gas (which is often the case in MEMS) with the elastic structure is not considered using molecular approach. For a gas model description of MEMS, the Knudsen number $\mathrm{Kn}$ (the ratio of mean free path of molecules to specific geometrical dimension) is a key dimensionless parameter. In such devices, the Knudsen number at standard temperature and pressure is large due to the small characteristic size. Continuum approach (Navier-Stokes equations) is applicable only at small Knudsen numbers and under special boundary conditions, taking into account small deviations from local equilibrium. For a slip-flow regime within $0.001<\mathrm{Kn}<0.1$ Navier-Stokes (NS) equations are applicable to the flow, but slippage on the border between the gas and the surface of the body should be taken into account, while for $0.1<\mathrm{Kn}<10.0$ the NS equations are no longer valid.

In the present work, a gas flow in a microchannel with an elastic thin cantilever clamped to the bottom of the microchannel is considered for $\mathrm{Kn}=0.05$ and different pressure ratios. The geometrically nonlinear version of the Euler-Bernoulli beam theory is used to model the cantilever oscillations and the gas flow is simulated by the DSMC method. The beam displacements and velocities are synchronized in both models. The used approach has been presented in [10]. Up to our knowledge, it is a first study where a gas flow has been simulated by the molecular approach (Direct Simulation Monte Carlo (DSMC) method) interacting with an elastic structure. The approach guarantees full coupling of gas-elastic beam interaction for unlimited time of simulation

\section{A model of a pressure driven gas flow in a microchannel with an obstacle}

Fig. 1 presents schematically the problem considered in this work. Two-dimensional pressure driven rarefied gas flow, which interacts with an obstacle is considered. The obstacle is an elastic beam fixed to the bottom wall. Pressure driven channel flow is well-studied problem. It represents two-dimensional flows of a viscous fluid in a space between two infinite parallel plates. The fluid is moving due to pressure difference at the channel inlet and outlet. Placing an elastic beam in the channel turns this flow into a complex non-linear problem.

The hybrid method for modelling of interactions of rarefied gas flow and elastic obstacle is presented in [10]. This is the first fluid structure interaction method that uses molecular approach to model the fluid. However, the methods used to analyse the problem are briefly presented below. 


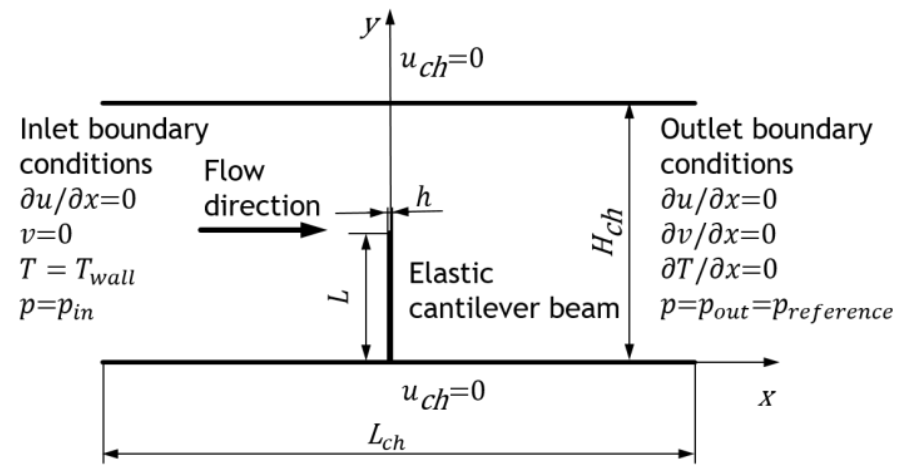

Fig. 1. A geometrical scheme of the problem

\subsection{The model of the elastic obstacle}

The elastic element inserted in the channel has been modelled as a cantilever beam. The geometrically non-linear version of the Euler-Bernoulli beam theory is used to describe the beam motion.

The equations of motion of the beam, which consider large displacements can be presented by the following equations [11]:

$$
\begin{gathered}
\frac{\partial N}{\partial y}+\rho A \frac{\partial^{2} u}{\partial t^{2}}=0, \\
\frac{\partial^{2} M}{\partial y^{2}}+c_{\mathrm{d}} \frac{\partial w}{\partial t}+N \frac{\partial^{2} w}{\partial y^{2}}+\rho A \frac{\partial^{2} w}{\partial t^{2}}=p(y, t), \quad 0<y<L,
\end{gathered}
$$

where $N$ and $M$ are the generalized stresses

$$
N=\int_{-h / 2}^{h / 2} \sigma_{y} d z, \quad M=\int_{-h / 2}^{h / 2} \sigma_{y} z d z .
$$

In (1) $u$ is the displacement along $x$ axis (longitudinal displacement), $w$ is the displacement along $y$ axis (transverse beam displacement), $\rho$ is the density of the material of the beam, $p(y, t)$ is the transverse loading of the beam, $A=b h$ is the area of the beam cross-section, $h$ is the thickness and $b$ is the width of the beam, $t$ is the time and by $c_{\mathrm{d}}$ the material damping coefficient. The length of the beam is $L$.

Considering the geometrical relationship:

$$
\varepsilon_{y}=\frac{\partial u}{\partial y}+\frac{1}{2}\left(\frac{\partial w}{\partial y}\right)^{2}
$$

and the constitutive equation

$$
\sigma_{y}=E \varepsilon_{y}=E\left[\frac{\partial u}{\partial y}+\frac{1}{2}\left(\frac{\partial w}{\partial y}\right)^{2}\right],
$$

the equations of motion of the beam can be transformed in: 


$$
E I \frac{\partial^{4} w}{\partial x^{4}}+c_{\mathrm{v}} \frac{\partial w}{\partial t}+\rho A \frac{\partial^{2} w}{\partial t^{2}}=p(y, t)+G_{L}(y, t)
$$

where

$$
G_{L}(y, t)=\frac{E I}{L}\left(\frac{6}{h^{2}} \int_{0}^{L}\left(\frac{\partial w}{\partial y}\right)^{2} d y\right) \frac{\partial^{2} w}{\partial y^{2}}
$$

and $c_{\mathrm{v}}=\frac{c_{\mathrm{d}}}{E I}$.

In (3) and (4) by $E$ the elastic Young's modulus is denoted and $I$ is the geometrical moment of inertia of the beam cross-section. In the case of rectangular cross-section of the beam it is $I=b h^{3} / 12$.

The boundary conditions for the elastic obstacle are for a cantilever (clamped at the one end and free at the tip ) [11]:

$$
\begin{gathered}
\text { for } y=0: w(0, t)=0, \quad \frac{\partial w(0, t)}{\partial y}=0, \\
\text { For } y=L: \frac{\partial^{2} w(0, t)}{\partial y^{2}}=0, \quad \frac{\partial^{3} w(0, t)}{\partial y^{3}}=0 .
\end{gathered}
$$

As far, as the considered case concerns micro objects and the dimensions of the channel and the beam are very small, it is necessary to use dimensionless variables in the numerical computations.

The following dimensionless variables are introduced:

$$
\bar{y}=y / L, \quad \bar{w}=w / L, \quad \bar{t}=t c / L, \quad c=\sqrt{E / \rho} .
$$

Then (4) and (5) are transformed in:

$$
\frac{\partial^{4} \bar{w}}{\partial \bar{y}^{4}}+c_{\mathrm{v}} \frac{\partial \bar{w}}{\partial \bar{t}}+\alpha^{-1} \frac{\partial^{2} \bar{w}}{\partial \bar{t}^{2}}=p_{1}+\bar{G}_{L}(y, t)
$$

$$
p_{1}=\frac{p(\bar{y}, t) L^{3}}{E I}, \quad \bar{G}_{L}=\frac{1}{2}\left(\int_{0}^{1}\left(\frac{\partial \bar{w}}{\partial \xi}\right)^{2} d \xi\right) \frac{\partial^{2} \bar{w}}{\partial \bar{y}^{2}}, \alpha=\frac{12 L^{2}}{h^{2}} .
$$

In the following equations, for convenience, the bars over the dimensionless variables will be omitted.

Equation (8) has to be coupled with the gas flow solution. For this purpose, a reduced model of the beam is developed here.

According to Galerkin procedure, the solution is sought by series

$$
w(y, t)=\sum_{n=1}^{N_{\mathrm{f}}} w_{n}(y) q_{n}(t),
$$

where $w_{n}(\mathrm{y})$ are space functions representing vibration modes which should satisfy geometrical boundary conditions, $q_{n}(\mathrm{t})$ are time functions, and $N_{\mathrm{f}}$ is the number of modes in the expansion. The natural modes of vibrations $w_{n}$ have the expression

$$
w_{n}(y)=A_{n}\left[\sin \beta_{n} y-\sinh \beta_{n} y-B_{n}\left(\cos \beta_{n} y+\cosh \beta_{n} y\right)\right],
$$

where the following notations are used: 


$$
\beta_{n}^{4}=\omega_{n}^{2} \alpha, \quad B_{n}=\frac{\sin \beta_{n}+\sinh \beta_{n}}{\cos \beta_{n}+\cosh \beta_{n}},
$$

and $\omega_{n}$ are the dimensionless natural frequencies of the linear Euler-Bernoulli beam obtained from the frequency equation

$$
\cos \beta \cosh \beta=-1 \text {. }
$$

Substituting (9) into partial differential Equation (4), multiplying it by the modal functions, integrating through the beam length, invoking the orthogonality condition and assuming the proportional damping the equation is transformed to a set of coupled ordinary differential equations of motion:

$$
\ddot{q}_{n}(t)+2 \xi_{n} \omega_{n} \dot{q}_{n}+\omega_{n}^{2} q_{n}(t)=F_{n}(t),
$$

where, $\xi_{n}$ are modal damping parameters and

$$
F_{n}(t)=\int_{0}^{1} w_{n}(y)\left[P(y, t)+G_{L}(y, t)\right] d y .
$$

In the present work, we have chosen the three modes reduction $\left(N_{\mathrm{f}}=3\right)$. The preliminary studies of the vibration behaviour of the beam subjected to step loading showed that the higher modes do not influence the response. On the other hand, using three modes reduction leads to manageable calculations to obtain the system of ordinary differential equations, which could be solved quickly and easily by the available software.

Using the three modes reduction the mathematical model of the vibrating beam takes the form:

$$
\begin{gathered}
\ddot{q}_{n}(t)+2 \xi_{n} \omega_{n} \dot{q}_{n}+\omega_{n}^{2} q_{n}(t)+D_{111}^{n} q_{1}^{3}+D_{222}^{n} q_{2}^{3}+ \\
+D_{333}^{n} q_{3}^{3}+D_{112}^{n} q_{1}^{2} q_{2}+D_{113}^{n} q_{1}^{2} q_{3}+D_{221}^{n} q_{1} q_{2}^{2}+ \\
+D_{223}^{n} q_{2}^{2} q_{3}+D_{331}^{n} q_{1} q_{3}^{2}+D_{332}^{n} q_{2} q_{3}^{2}+D_{123}^{n} q_{1} q_{2} q_{3}=F_{n}^{p}(t),
\end{gathered}
$$

Here

$$
D_{i j k}^{n}=0.5 \int_{0}^{1} w_{n} C_{i j k} d y, \quad i, j, k=1,2,3,
$$

and

$$
\begin{gathered}
C_{111}=I_{11} w_{1}^{\prime \prime}, C_{222}=I_{22} w_{2}^{\prime \prime}, C_{333}=I_{33} w_{3}^{\prime \prime}, \\
C_{112}=I_{11} w_{2}^{\prime \prime}+2 I_{12} w_{1}^{\prime \prime} \quad C_{113}=I_{11} w_{3}^{\prime \prime}+2 I_{13} w_{1}^{\prime \prime}, \\
C_{221}=I_{22} w_{1}^{\prime \prime}+2 I_{12} w_{2}^{\prime \prime}, \quad C_{223}=I_{22} w_{3}^{\prime \prime}+2 I_{23} w_{2}^{\prime \prime}, \\
C_{331}=I_{33} w_{1}^{\prime \prime}+2 I_{13} w_{3}^{\prime \prime}, \quad C_{332}=I_{33} w_{2}^{\prime \prime}+2 I_{23} w_{3}^{\prime \prime}, \\
C_{123}=2\left(I_{23} w_{1}^{\prime \prime}+I_{13} w_{2}^{\prime \prime}+I_{12} w_{3}^{\prime \prime}\right), \\
I_{i j}=\int_{0}^{1} w_{i}^{\prime} w_{j}^{\prime} d y .
\end{gathered}
$$


The nonlinear system of ordinary differential equations (14) was solved by the Gear's method [12].

\subsection{DSMC}

The Direct Simulation Monte Carlo (DSMC) method was proposed by B i r d [13] in the late 1960s. It is widely used molecular approach to simulate rarefied gas flow in upper layers of atmosphere and in different microsystems as MicroElectroMechanical Systems (MEMS), micropumps, flow sensors, etc.

DSMC uses some simplifications that make possible to reduce computational resources of molecular approach and fulfil the gap between molecular dynamics and continuum models. Real motion of the particles is split in two parts. The ballistic motion of all the particles and the interaction between the molecules without changing their positions. The first step is deterministic, while the collisions between particles are carried out in a stochastic manner. Other important characteristics of DSMC is that one simulator particle, for shortly particle, represents many real molecules or atoms. This assumption reduces significantly computational resources. DSMC fulfils the gap between molecular dynamics and continuum models where molecular dynamics requires impossibly enormous computational resources and continuum models are no longer valid. One can see a detailed summary of different collision models developed in the framework of the DSMC method [14]. The collision procedure used here is advanced Simplified Bernoulli Trial (SBT) scheme, [15]. SBT collision scheme obtains correct results for small number of particles per cell (see $[16,17])$ that makes it straightforwardly applicable to unstructured and continuously changing mesh as our case is.

DSMC uses Cartesian uniform mesh in computational domain and unstructured mesh near elastic beam, see Fig. 5, right part. This way we combine fast indexing of the particles in uniform mesh and calculation of flow past complex geometries with minimal slow down, as indexing of the particles in unstructured mesh requires much more computational resources. In cells of a basic mesh the Transient Adaptive Subcells (TAS) technique [18] is used to improve the DSMC spatial accuracy. 2D adaptive unstructured mesh near geometry was generated using Delaunay algorithm by mesh generator Gmsh [19] with $\mathrm{C}^{++}$API functions.

DSMC uses a hard-sphere model of monoatomic gas and the diffuse reflection boundary condition on the microchannel and the elastic beam walls.

\section{Validation}

\subsection{Verification of the beam model}

The suggested model of the geometrically nonlinear vibration of the beam has been checked by comparing the results obtained by the reduced model with theese obtained by the commercial program MSC NASTRAN. The loading equivalent to the one obtained from the gas pressure cannot be applied to the NASTRAN model and that is why a uniformly distributed along the beam length step loading was considered. It turned out that MSC Nastran could not calculate problems for structures with very 
small dimensions, which has been considered in our numerical examples (singularities arise). That is why the calculations have been performed for a beam with the geometrical dimensions 100 times bigger than the one considered in our numerical examples. The dimensions are $L=0.248 \mathrm{~m}, h=0.0248 \mathrm{~m}, b=0.0496 \mathrm{~m}$.

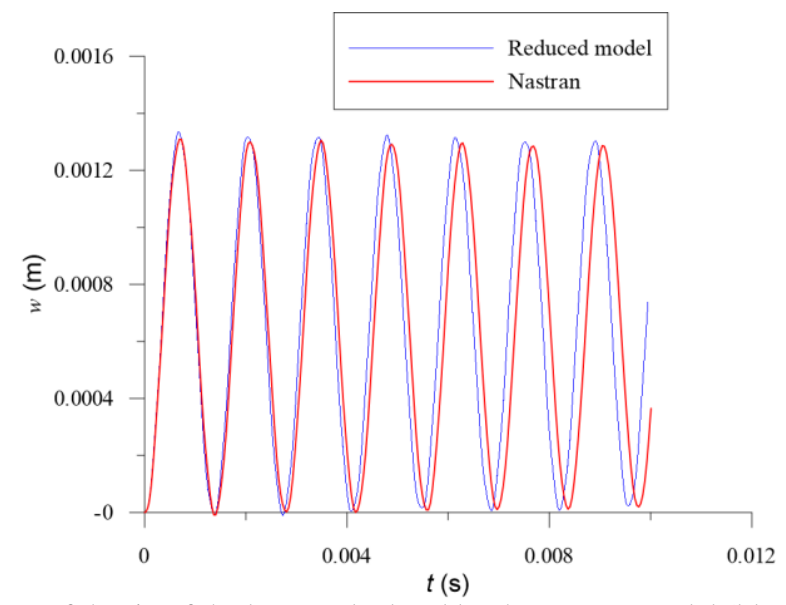

Fig. 2. The response of the tip of the beam calculated by the present model (blue colour) and FEM program MSC NASTRAN

The material properties were the same as those used in our calculations of the coupled problem: $E=1.128 \mathrm{MPa}$ and $\rho=8960 \mathrm{~kg} / \mathrm{m}^{3}$. The beam has been discretized by 40 linear beam elements ( 41 nodes). The applied load is $p=10 \mathrm{kN} / \mathrm{m}$. As can be seen in Fig. 2, the results obtained by the both models are very similar and the differences in amplitudes are very small. There is a small shift of the periods. This could be explained with the small differences in the natural frequencies calculated by our model (analytical ones) and the ones obtained by MSC NASTRAN (not shown here).

These results allow us to decide that our model describes with a sufficient accuracy the beam motion.

\subsection{DSMC validation of pressure driven flow in a long channel}

It is known that the results obtained by continuum and molecular approaches are still close at $\mathrm{Kn}=0.05$, and therefore the continuum approach can be used to validate the results obtained by DSMC. The result of DSMC with SBT collision scheme is compared with available analytical solution of viscous, compressible isothermal flow in a long microchannel [20], Navier-Stokes equations with velocity slip and temperature jump boundary conditions on channel walls, and DSMC with NoTime Counter (NTC) collision scheme [13] (see Fig. 3). Navier-Stokes equations are calculated by finite-volume method SIMPLE-TS [21]. The considered pressure ratio $\left(\mathrm{P}=p_{\text {in }} / p_{\text {out }}\right)$ is 3 , Knudsen number is 0.05 , and the channel aspect ratio (channel length to the channel height) is equal to 50. DSMC basic Cartesian uniform mesh is $2000 \times 40$ cells with a total number of $2 \times 10^{6}$ particles. TAS technique is applied at every basic Cartesian cell with average Particles Per Cell (PPC) 2. The result of DSMC with SBT shows excellent agreement with DSMC with NTC collision scheme. The comparison 
of DSMC results with continuum approaches (analytical solution and Navier-Stokes) shows excellent agreement. Similar expected differences between molecular and continuum models are pointed out in the works of other authors (for example, see [18]).

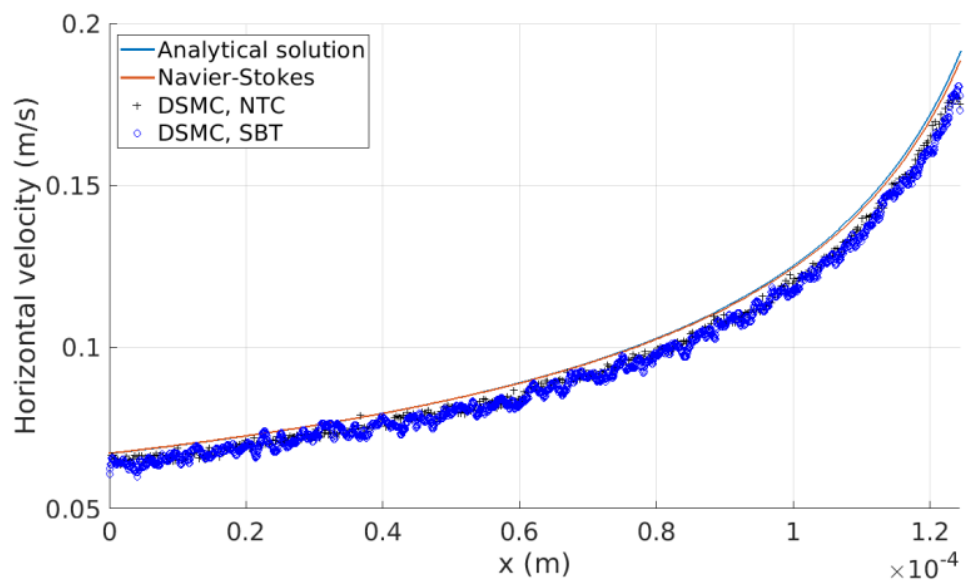

Fig. 3. Horizontal component of velocity along the centerline of the channel $y=H_{\mathrm{ch}} / 2$. Horizontal velocity profiles, from analytical solution [20], continuum model (Navier-Stokes) calculated by SIMPLE-TS method [21], DSMC NoTime Counter (NTC) collision scheme [13], DSMC SBT with TAS with PPC 2

\section{Results and discussions}

We consider a non-stationary pressure driven gas flow in a micro-channel with an elastic beam fixed to the bottom channel wall (Fig. 1). The initial condition is a gas at rest, the pressure of the gas in the channel is equal to the pressure at the outlet $p_{\text {out, }}$ and the gas temperature is equal to the temperature of the channel walls $T_{\text {wall }}$. At zero time $t=0 \mathrm{~s}$ the inlet is opened suddenly and the gas under pressure $p_{\text {in }}$ and temperature $T_{\text {in }}=T_{\text {wall }}$ enters the channel. We consider the transient and established gas flow. The characteristc length used in the calculations of the gas flow is equal to the elastic beam length. The length of the beam is $L=2.49 \times 10^{-6} \mathrm{~m}, h=0.1 L, b=2 h$, the height of the channel is $H_{\mathrm{ch}}=2 L$, and the channel length is $L_{\mathrm{ch}}=10 L$. Knudsen number is equal to 0.05 . We considered numerical examples with two values of the pressure ratio. DSMC basic Cartesian uniform mesh is $400 \times 80$ cells and the number of cells (elements) of unstructured mesh around the elastic beam varies around 2500. A total number of particles for pressure ratio $P=1.5$ and 3 is $4.8 \times 10^{5}$ and $8.2 \times 10^{5}$, respectively.

In Fig. 4 (left part) are shown horizontal velocity, vertical velocity, pressure, and temperature fields of a rarefied gas when flow is established. One can see that cantilever plays a role of a bend that separates the channel, and turns the pressure driven gas flow to be similar to a gas flow through a short nozzle. The gas flow enters the channel and the pressure in front of the beam is close to the inlet pressure. After the tip of the beam, the velocity accelerates due to significant pressure drops, the gas rarefies, and the temperature drops. Fig. 4 (right part) shows used mesh in DSMC 
rarefied gas modelling. Basic mesh is a Cartesian uniform mesh. The unstructured mesh near elastic beam captures irregular form geometry of the beam. In this way we have used Cartesian mesh to do fast calculations and a slower unstructured mesh near cantilever to model the flow near common geometries. The red line is neutral line of the cantilever that is modelled from the Cantilever's code.

These results have been obtained for $P=3$. These for $P=1.5$ are similar in qualitative sense and that is why they are not shown here. More essential influence of the pressure difference can be observed on the beam vibration.

In the numerical calculations, two models of the cantilever have been used - a linear model using Small Deflection Beam Theory (SDBT) and nonlinear model using Large Deflection Beam Theory (LDBT).

The loading arising at the tip of the beam in time for $P=1.5$ is shown in Fig 5. It is clearly seen that the loading has a stochastic character. It is natural because the molecular approach (DSMC) models the movements of the particles in the rarefied gas flow.

The response of the beam is shown in Fig. 6. The stochastic loading leads to non-periodic vibration of the beam. Essential differences can be observed in the results obtained by SDBT and LDBT. Generally, it could be concluded that the amplitude of vibration obtained by SDBT are a little larger than the obtained by LDBT. This is explicable from theoretical point of view because the large deflection beam theory considers the axial force, which arises in the beam axis. This increases frequencies and decreases amplitudes of vibration. The small figure inserted in the large figure presents the behaviour of the tip of the beam at the beginning of the process. It can be seen that there is a period when the beam almost does not vibrate (vibrations are with very small amplitudes in comparison with these after the initial period). The reason is that initially (at transient period) compression wave propagates from the channel inlet due to entrance of the gas in a channel with higher pressure $\left(p_{\text {in }}\right)$ than the pressure in channel $\left(p_{\text {out }}\right)$ at the initial moment. The wave needs time to propagate from the channel inlet to the elastic beam. When the wave reaches the elastic beam, it reflects partially (lower part) and propagates backword to the channel inlet and partially (upper part) continues to the channel outlet.

This phenomenon is more clearly seen in Fig. 8 where the time history diagram of the tip of the beam subjected to a pressure ratio $P=3$ is shown. The loading on the tip of the beam in this case is shown in Fig. 7. It has a stochastic character as in the previous case. Here the interaction of compression wave with the elastic beam is more clearly seen which arises at the beginning of the process and leads to large values of the pressure on the beam for a short period. The considered gas phenomenon corresponds to water hammer phenomenon. That is why the displacements of the tip of the beam at the transient period are much larger than the vibration after it $\left(t>2.28 \times 10^{-7} \mathrm{~s}\right)$. The vibration of the beam at the initial period are small, periodic and regular. The stochastic character of the response manifests at the later periods. In this case also the amplitudes of vibration obtained by SDBT are a little larger than the ones obtained by LDBT. 

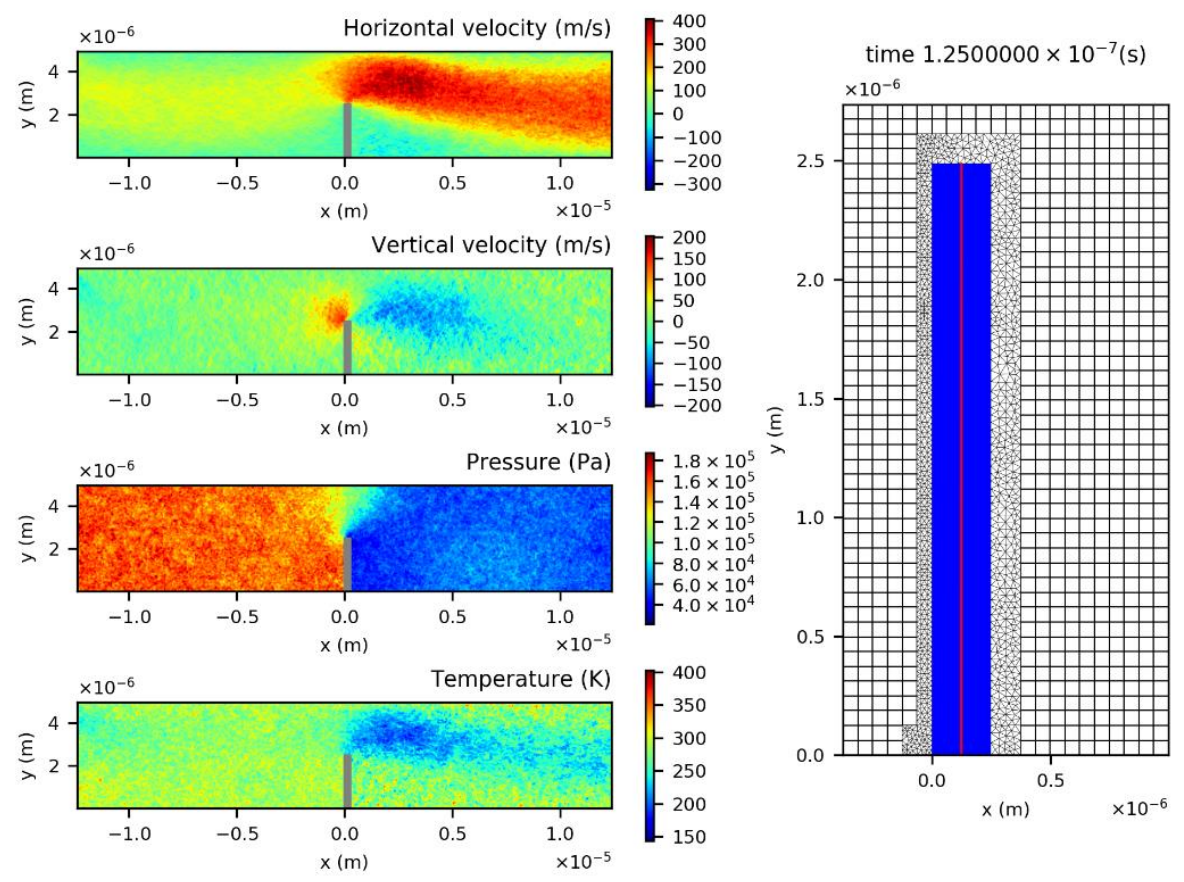

Fig. 4. Horizontal velocity, vertical velocity, pressure, and temperature fields, from top to bottom (left part) and deflections along the beam length, Cartesian basic mesh, and unstructured mesh near the beam (right part) for $P=3$ and $\mathrm{Kn}=0.05$

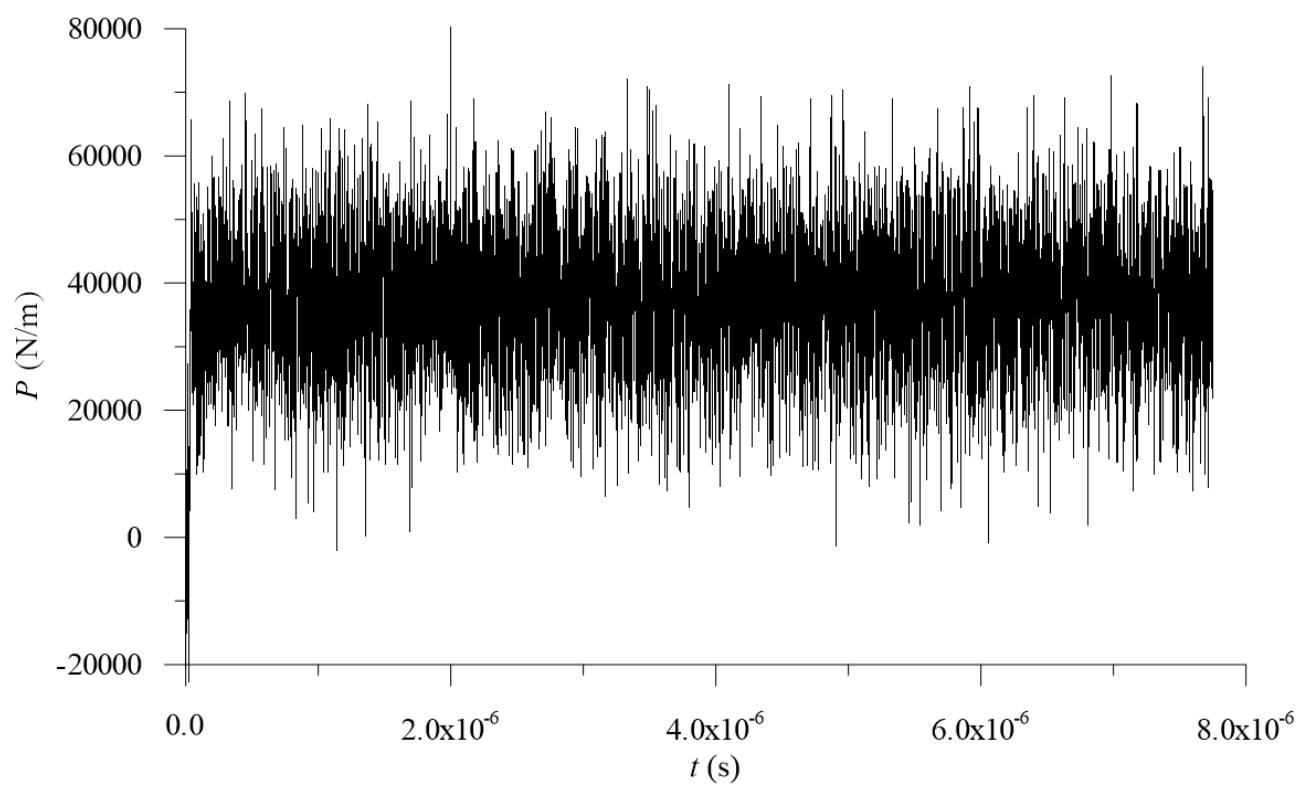

Fig. 5. The loading at the top of the beam for $P=1.5$ in time 
The obtained results demonstrate that the developed computer codes and suggested algorithms are capable to study the fully coupled problem of the interaction between pressure driven flow of rarefied gas in micro channel and an elastic obstacle. Up to our knowledge, the present study is the first attempt in this direction. This approach can be used for design and analysis of highly technological devices.

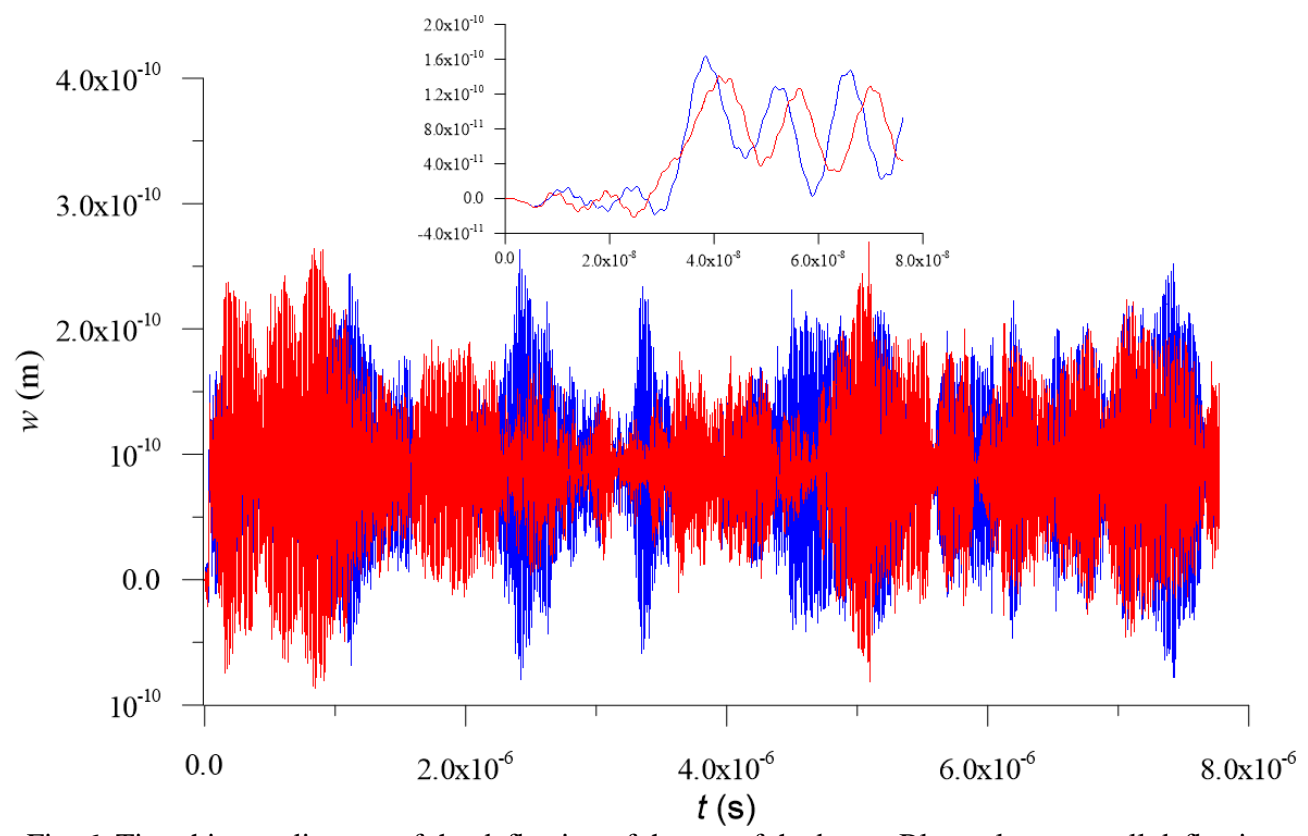

Fig. 6. Time-history diagram of the deflection of the top of the beam. Blue colour - small deflection beam theory. Red line - large deflection beam theory and $P=1.5$

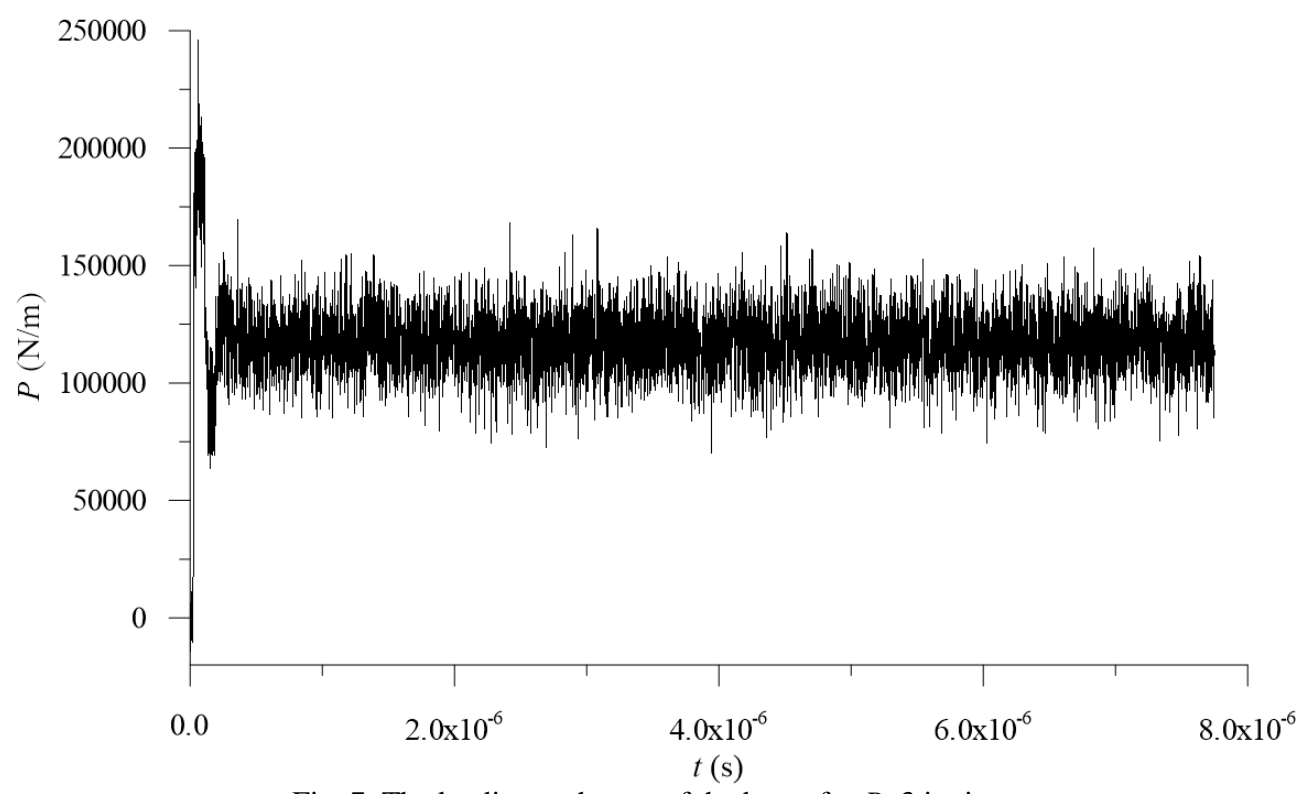

Fig. 7. The loading at the top of the beam for $P=3$ in time 


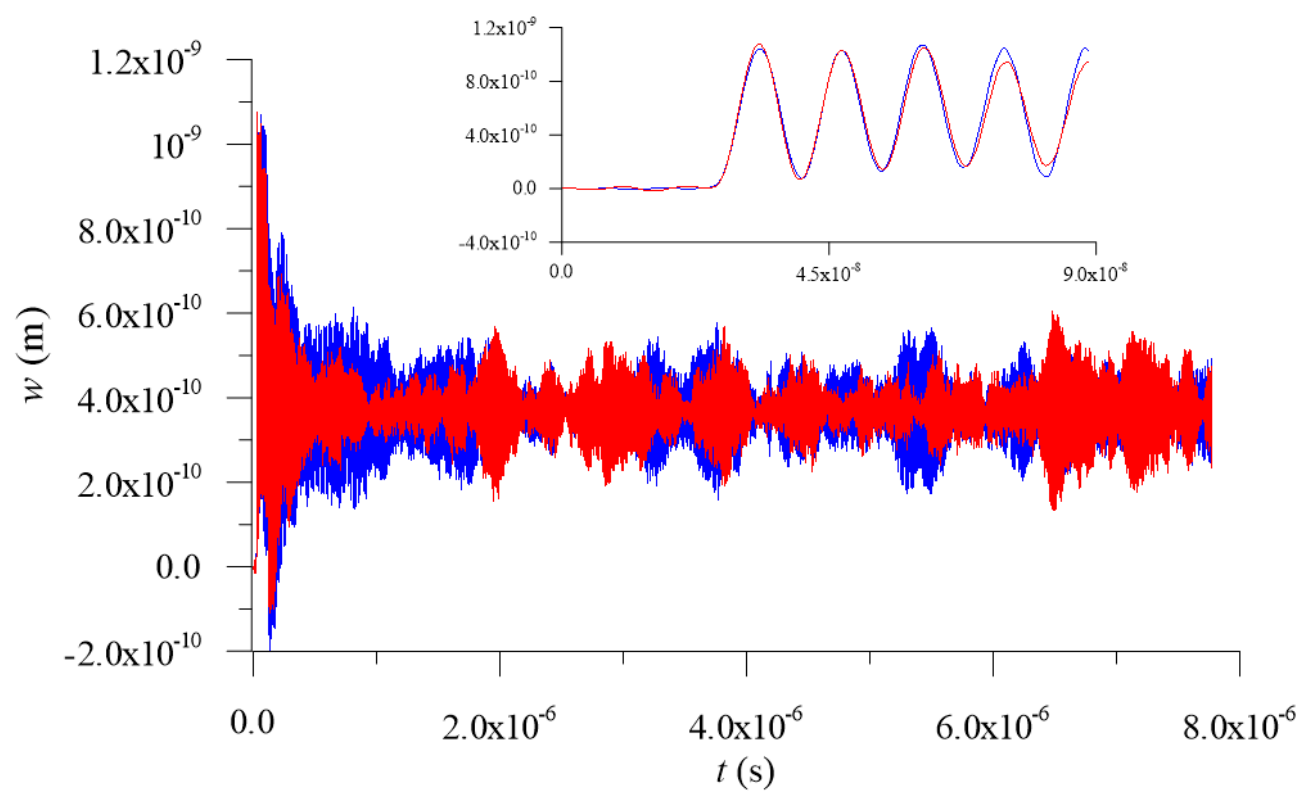

Fig. 8. Time-history diagram of the deflection of the top of the beam. Blue colour - small deflection beam theory. Red line - large deflection beam theory. $P=3$

Acknowledgments: This work has been accomplished with the financial support of Grant KP-06-N32/6, 2019 from the Bulgarian research fund and the Ministry of Education and Science under Grant No BG05M2OP001-1.001-0003, provided by the SESG Operational Program (2014-2020) and co-financed by the European structural and investment funds.

\section{References}

1. P a i d o u s s i s, M. Fluid-Structure Interactions. Second Edition. 2013.

2. Martin, J. M., H. K. Fathy, B. H. Housto n. Dynamic Simulation of Atomic Force Microscope Cantilevers Oscillating Inliquid. - J. Appl. Phys., Vol. 104, 2008, 044316.

3. Col e, D. G., R. L C l a r k. Fluid-Structure Interaction in Atomic Force Microscope Cantilever Dynamics and Thermal Response, 6. - J. Appl. Phys.,Vol. 101, 2007. 10.1063/1.242972.

4. G r e e n, C.P., J. E. S a d e r. Frequency Response of Cantilever Beams Immersed in Viscous Fluids near a Solid Surface with Applications to the Atomic Force Microscope. - J. Appl. Phys., Vol. 98, 2005, 114913

5. Corneli s, A., E. V a n, J. E. S a d e r. Frequency Response of Cantilever Beams Immersed in Viscous Fluids with Applications to the Atomic Force Microscope: Arbitrary Mode Order. J. Appl. Phys., Vol. 101, 2007, 044908.

6. V a n R i j, J., T. H a r m a n, T. A m e e 1. Slip Flow Fluid-Structure Interaction. - International Journal of Thermal Sciences, Vol. 58, 2012, pp. 9-19.

7. $\mathrm{Ch}$ a k r a b o r ty, D., et al. Fluid-Structure Interaction in Deformable Microchannels. - Physics of Fluids, Vol. 24, 2012, No 10, p. 102002.

8. P a n, F., J. K u b i, J. Ch e n. Numerical Simulation of Fluid-Structure Interaction in a MEMS Diaphragm Drop Ejector. - J. Micromech. Microeng., Vol. 12, 2002, No 1, 70.

9. B a u d i 11 e, R., M. E. B i a n c o 1 i n i. A General Approach for Studying the Motion of a Cantilever Beam Interacting with a 2D Fluid Flow. - Interact. Multisc. Mech., Vol. 1, 2008, pp. 449-465.

10. Shterev, K., E. Manoach, S. Ste f a nov. Hybrid Numerical Approach to Study the Interaction of the Rarefied Gas Flow in a Microchannel with a Cantilever. - International Journal of Non-Linear Mechanics, Vol. 117, 2019, 103239.

11. M e i r o vit c h, L. Fundamentals of Vibrations. McGraw-Hill, 2001. 
12. G e a r, C. W. Numerical Initial Value Problems in Ordinary Differential Equations. Upper Saddle River, NJ, USA, Prentice Hall PTR. 1971.

13. B i r d, G. A. Molecular, Gas Dynamics and the Direct Simulation of Gas Flows. Oxford, Clarendon Press, 1994

14. R o o hi, E., S. S t e f a n o v. Collision Partner Selection Schemes in DSMC: From Micro/Nano Flows to Hypersonic Flows. - In: Physics Reports. Vol. 656. 2016, pp. 1-38.

15. S t e f a n o v, S. K. On DSMC Calculations of Rarefied Gas Flows with Small Number of Particles in Cells. - SIAM Journal on Scientific Computing, Vol. 33, 2011, No 2, pp. 677-702.

16. T a h e ri, E., E. R o oh i, S. S te f a n o v. On the Convergence of the Simplified Bernoulli Trial Collision Scheme in Rarefied Fourier Flow. - Physics of Fluids, Vol. 29, 2017, 062003.

17. R oohi, E., et al. A Generalized Form of the Bernoulli Trial Collision Scheme in DSMC: Derivation and Evaluation. - Journal of Computational Physics, Vol. 354, 2018, pp. 476-492.

18. B i r d, G. A. Visual DSMC Program for Two-Dimensional and Axially Symmetric Flows. - In: The DS2V Program User'S Guide. Version 3.8. 2006. Sydney NSW 2000, Australia, G. A. B. Consulting Pty. Ltd.

19. G e u z a i n e, C., J.-F. R e m a c l e. Gmsh: A 3-D Finite Element Mesh Generator with Built-in Preand Post-Processing Facilities. - International Journal for Numerical Methods in Engineering, Vol. 79, 2009, No 11, pp. 1309-1331.

20. A r ki l i c, E. B., M. A. S c h m i d t, K. S. B r e u e r. Gaseous Slip Flow in Long Microchannels. - Journal of Microelectromechanical Systems, Vol. 6, 1997, pp. 167-178.

21.S h t e re v, K. S., S. K. S t e f a n o v. Pressure Based Finite Volume Method for Calculation of Compressible Viscous Gas Flows. - Journal of Computational Physics, Vol. 229, 2010, pp. 461-480.

Received: 15.09.2020; Second Version: 20.10.2020; Accepted: 23.10.2020 